\title{
Center of mass perception and inertial frames of reference
}

\author{
GEOFFREY P. BINGHAM and MICHAEL M. MUCHISKY \\ Indiana University, Bloomington, Indiana
}

\begin{abstract}
Center of mass perception was investigated by varying the shape, size, and orientation of planar objects. Shape was manipulated to investigate symmetries as information. The number of reflective symmetry axes, the amount of rotational symmetry, and the presence of radial symmetry were varied. Orientation affected systematic errors. Judgments tended to undershoot the center of mass. Random errors increased with size and decreased with symmetry. Size had no effect on random errors for maximally symmetric objects, although orientation did. The spatial distributions of judgments were elliptical. Distribution axes were found to align with the principle moments of inertia. Major axes tended to align with gravity in maximally symmetric objects. A functional and physical account was given in terms of the repercussions of error. Overall, judgments were very accurate.
\end{abstract}

Perceptual information used to control and coordinate object manipulation can be obtained either haptically or visually. As soon as contact with an object is established, haptic information is available. In preparation for grasping before contact, the location for a grasp on an object must be determined using visual information. The intended grasp locus along with perceived object shape and size determine the direction of the reach, the orientation of the hand, and the relative extension of the fingers and thumb (Jeannerod, 1984; Jeannerod, 1988; Wing \& Fraser, 1983). However, the intended grasp locus also depends on the goal in the specific task being performed.

The basic intent in grasping a detached object is to control the position or trajectory of the object in free space. This requires that the object's weight be supported by the hand. Occasionally, an object is supported by placing the hand underneath the object without enclosing it. However, the manipulation allowed by this posture is very limited. At the other extreme, an object's weight can be sustained solely by a friction force between hand and object surfaces. This force is a function of the roughness of hand and object surfaces and of the forces applied perpendicularly forcing the surfaces together. To fix an object in the hand, equal and opposite forces must be applied by the hand to surfaces on opposite sides of the object. Thus, for equilibrium, there must be at least two contact forces, and if there are only two, these must be colinear and oppositely directed. ${ }^{1}$

This work was supported by the Institute for the Study of Human Capabilities at Indiana University, by an Indiana University Summer Faculty Fellowship award to G.P.B., and by National Science Foundation Grant BNS-9020590. Portions of the research were reported previously (Bingham \& Muchisky, 1990, 1991a, 1991b). Send all correspondence to G. P. Bingham, Department of Psychology, Indiana University, Bloomington, IN 47405.
A grasp in which finger and thumb pads contact and pinch an object is called a "precision grasp" in Napier's widely used classification (Napier, 1980). Precision grasps are used with great frequency in assembly tasks where small parts must be manipulated for precise placement. The stability of such grasps is important because objects in an unstable grasp may be difficult to put in place and dropped parts may be broken or lost. On the other hand, a momentary instability can be used to allow an object to rotate within the grasp to a desired stable position.

We will refer to the line between the thumb and finger along which contact forces are directed in a precision or pinching grasp as the "opposition axis" (Iberall, Bingham, \& Arbib, 1986). The stability achieved in a simple precision grasp at equilibrium depends on the location of the opposition axis with respect to the center of mass of an object. ${ }^{2}$ The grasp can be stable, unstable, or neutrally stable. Grasping an object above its center of mass yields a stable equilibrium; if perturbed, the object returns to its original orientation when the perturbing force is removed. This configuration allows passive maintenance of posture and is convenient, for instance, in keeping a cup of hot coffee from spilling. Grasping below the center of mass yields unstable equilibrium; when perturbed away from the equilibrium position, the object continues to rotate toward the stable configuration with the center of mass located below the opposition axis. An unstable grasp may be desirable in one-handed manipulation to achieve passive changes in orientation. Finally, grasping an object so as to pass the opposition axis through the center of mass yields neutral stability. The object exhibits no preferred orientation about the opposition axis and will remain in any given posture in which it is placed. A neutrally stable grasp can be useful in precision positioning where the orientation of an object must be carefully controlled and adjusted during placement. This posture also might be de- 
sirable in situations where the final desired orientation is not apparent at the moment of initial grasping.

As shown by Iberall et al. (1986), who observed manual behaviors in assembly tasks, the center of mass is frequently used to determine the location of a grasp. They videotaped participants performing tasks while seated at a table. Participants used either one or two hands with or without vision. Two tasks were used: in one, participants assembled and disassembled a tower made from large plastic blocks; in the other, they assembled and disassembled a meat grinder weighing slightly more than $2 \mathrm{~kg}$, and consisting of six irregularly shaped and relatively heavy parts. Particularly with the meat grinder, participants were observed to establish precision grasps at centers of mass (an S-shaped crank handle was usually grasped in this way, for instance, in attaching it to the end of a screw-shaped blade). With vision, the desired location of the grasp was achieved on first contact. ${ }^{3}$ Further, the passive manipulation of objects by grasping below the center of mass was often observed in the onehanded mode, and sometimes also in the two-handed situation if the other hand was momentarily occupied in holding or supporting other parts.

If, as suggested by these observations, people can locate the center of mass in objects using only visual information, then what is that information? The center of mass is, obviously, a mass-related or dynamic property of objects. Such properties are often referred to as "nonvisual" (Quinlan, 1991, p. 238) or "hidden" (Runeson \& Frykholm, 1983), with concurrent recognition, nevertheless, that perceptual access to them is essential for the control and coordination of actions. The problem is that dynamic properties are described in units of mass in addition to those of length and time whereas patterns detectable by the perceptual systems involve only lengths and times. In the context of events, visual information about dynamics can be mapped through resulting motions into spatiotemporal optical patterns that can be detected by the perceptual apparatus (see, for instance, Bingham, 1987a, 1987b, in press; Bingham, Rosenblum, \& Schmidt, 1993; Bingham, Schmidt, \& Rosenblum, 1989; Bingham, Schmidt, Turvey, \& Rosenblum, 1991; Kugler, 1986; Kugler \& Turvey, 1987; Pittenger, 1985, 1990; Riccio, Martin, \& Stoffregen, 1992; Runeson, 1977; Runeson \& Frykholm, 1981, 1983; Runeson \& Vedeler, 1993; Solomon, Turvey, \& Burton, 1988, 1989b; Todd \& Warren, 1982; Warren, Kim, \& Husney, 1987). The center of mass might well be specified by object trajectories: in projectile motion, an object's center of mass follows a parabolic path while the remaining portions of the object follow more complex paths composed of a parabolic component plus a component of rotation around the center of mass.

However, before being grasped, objects often lie unmoving on support surfaces. If visual information about the center of mass is available in such situations, then that information must lie in the geometric properties of an object which would map, in turn, into properties of the optic array or optic flow. The distinction between informative object properties and corresponding properties comprising information in optical pattern is important. Although the informative object properties might be spatial or static, the corresponding optical properties typically would be spatiotemporal because the observer would be in motion even if the object was not. Our investigation focused on the object properties that might provide information about the center of mass. We leave the study of corresponding information in optical flows for future investigations.

In general, constant mass density is required if shape is to provide information about mass distribution. Singlematerial composition ensures nearly constant mass density and is usually recognizable. Fortunately, single-material objects are relatively common, and include the majority of natural objects and a large proportion of manufactured objects, such as those made from wood, soap, plastic, metal, ceramics, stone, glass, and organic fibers. The perception of the center of mass in objects manufactured from more than one material is necessarily more difficult. However, depending on their intended use, objects manufactured from composite materials are often designed so that the center of mass is specified by geometry-the center of mass of the first author's rectangularly shaped electric razor, for instance, lies at its geometric center.

The center of mass is a symmetry property of objects (Becker, 1954; Sears, Zemansky, \& Young, 1986). It is the point around which the mass distribution is balanced. Assuming constant mass density, the center of mass must fall on any axes of reflective symmetry in an object. The axis that divides an isosceles triangle into two right triangles is an axis of reflective symmetry. Three non-coplanar axes of reflective symmetry uniquely specify the location of the center of mass in an object. Detection of reflective symmetry could be a fundamental means of identifying the center of mass. If so, a larger number of axes of reflective symmetry should increase accuracy in locating the center of mass. Might accuracy improve with increases in the number of axes above three (or in planar objects, above two)?

Reflective symmetry can be defined not only with respect to an axis or line through which object points are reflected, but also with respect to a point. This is called radial symmetry (see Grünbaum \& Shephard, 1989; Lord \& Wilson, 1986; Rosen, 1982; Shubnikov, 1964; and Weyl, 1921, for various taxonomies of symmetry). An equilateral triangle has three axes of reflective symmetry but does not have radial symmetry, whereas a parallelogram has no axes of reflective symmetry but does have radial symmetry. In objects of constant mass density, the center of mass must lie at the focus of radial symmetry. Is the center of mass located more accurately in objects with radial symmetry?

A third type of symmetry is rotational symmetry. This is counted or indexed by the number of times selfcongruence occurs with a rotation through $360^{\circ}$. A parallelogram is self-congruent twice, at $180^{\circ}$ and $360^{\circ}$, while a square is self-congruent four times, at $90^{\circ}, 180^{\circ}, 270^{\circ}$, and $360^{\circ}$, right and isosceles triangles are only self- 
congruent once, at $360^{\circ}$, and an equilateral triangle is selfcongruent three times, at $120^{\circ}, 240^{\circ}$, and $360^{\circ}$. Might greater rotational symmetry yield greater accuracy in locating the center of mass?

Orientation is potentially relevant because axes of reflective symmetry have been reported to be recognized more readily when parallel to gravity (Rock, 1973). In Rock's studies, a reflective axis was recognized increasingly less often as it was rotated farther from a vertical orientation. Might accuracy in locating the center of mass increase when reflective symmetry axes are parallel to gravity?

The size of an object might affect accuracy in determining center of mass location. Error in judging the center of mass might increase in proportion to its distance to the defining outline of the shape. On the other hand, this tendency might be mitigated by increasing symmetry. The existence of axes of reflective symmetry or of a locus of radial symmetry could make distance to the outline less relevant (or perhaps alter its role). Might shape and size interact in determining the accuracy of judgments of the center of mass?

\section{EXPERIMENT 1}

We focused on tasks requiring precision grasps with neutral stability so that participants were required to target the center of mass in their grasping. We used planar objects of a single material-namely, shapes cut from a wooden board. Only two coordinates were required to locate pinching grasps in terms of a single point on the object's planar surface. Judgments were expressed by using tongs to grasp the objects. The tongs allowed a determination of accuracy in perception beyond that required by characteristics of the hand. We manipulated three geometric properties: shape, orientation, and size. Symmetry properties of objects were manipulated by varying object shape to include shapes with or without radial symmetry and shapes with increasing numbers of reflective symmetry axes (and, concurrently, shapes with increasing amounts of rotational symmetry). Objects of three different sizes in each of seven different shapes were presented in three different orientations.

\section{Method}

Participants. Thirty undergraduates at Indiana University participated in partial fulfillment of an introductory psychology course requirement. Twenty-four were female and 6 were male. All had normal or corrected-to-normal vision and reported no motor disabilities.

Apparatus. A set of seven planar objects was designed to vary the amount of symmetry. Shapes were cut from 1-cm-thick plywood. The number of axes of reflective symmetry ranged from zero to four. Rotational symmetry in the plane of the objects varied from one to four loci of self-congruence within a $360^{\circ}$ rotation. Finally, four of the shapes possessed radial symmetry. The shapes and symmetries of the objects are shown in Figure 1 (the quadrilateral was used only in Experiment 2). Each of these planar shapes was created in three sizes (in $\mathrm{cm}^{2}$ ) as follows: right triangle: $75,100,163$; isosceles triangle: $74,100,140$; parallelogram: $66,100,285$; rect-

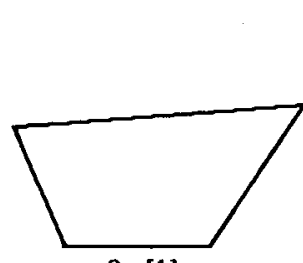

$0[1]$

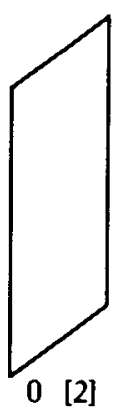

RS

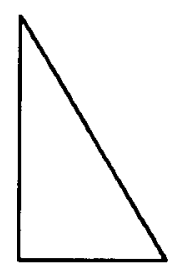

0 [1]
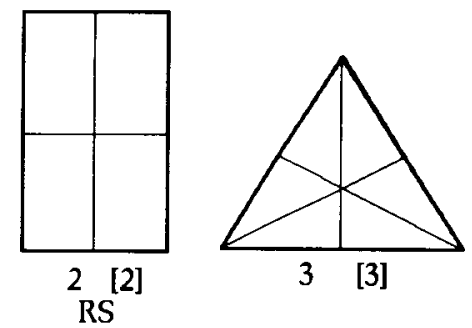

3 [3]

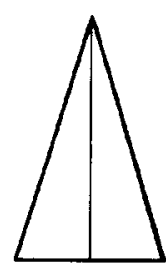

1 [1]

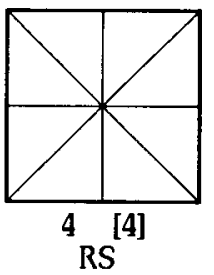

Figure 1. Shapes used in Experiments 1 and 2. The number and location of reflective symmetry axes are shown as well as (in brackets) the frequency of congruence in a $360^{\circ}$ rotation, the latter indicating the amount of rotational symmetry. Shapes that possess radial symmetry are indicated as RS.

angle: $100,113,144$; equilateral triangle: $92,100,149$; square: $72,100,165$. The largest set still allowed the tongs to reach far past the centers of the figures from almost any direction in the plane of the objects.

The objects were presented to each observer with the plane of the figure parallel to gravity. Objects were held upright in a transparent spring-loaded clamp affixed to a wooden base, as shown in Figure 2. The side of the figure facing the subject was an unfinished smooth wood surface. The side facing the experimenter had polarcoordinate paper attached to it with the origin of the coordinates fixed at the center of mass. The units were millimeters. The Archimedean method was used to determine the location of the center of mass in each object, which was suspended alternatively from two different points along its perimeter. A plumb line was hung from each point in turn and marked on the object. The intersection of the two lines marked the location of the center of mass. The tongs used to express judgments were held and manipulated in one hand like a large pair of scissors. The point of the tongs that contacted the surface viewed by the observer was padded to prevent indentation of the surface.

Procedure. The observers were asked to judge where they felt the "stable point" was. We explained that this term referred to the point where an object would remain stable without rotating about the point of contact when held upright with the thumb and index finger. Furthermore, if the object were to be rotated to another orientation, it would remain in the new orientation in which it had been placed. This was demonstrated using a different object from those used in the judgment trials. The term "stable point" was employed to avoid using the word "center" in the task description, and be- 


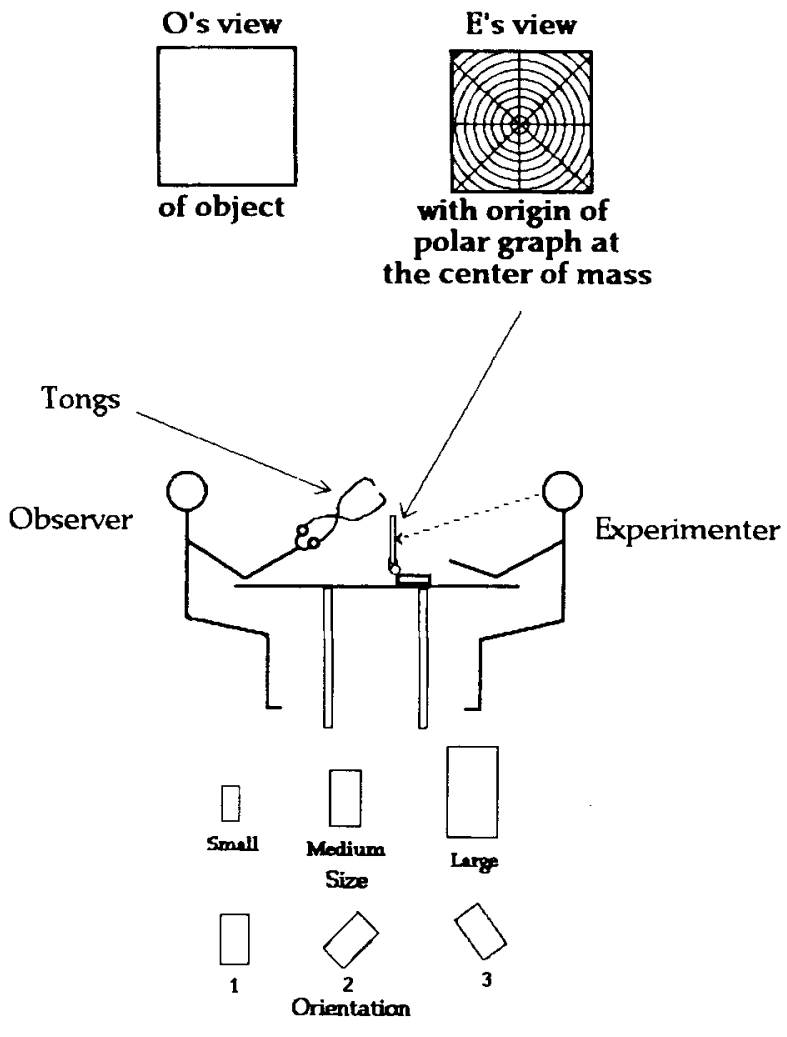

Figure 2. Presentation of Experiments 1 and 2.

cause stability was the disposition that we wished our observers to achieve in grasping. During each trial, the observers were asked to close their eyes while the object in the clamp was changed. In this way, they were prevented from obtaining information about the center of mass by witnessing the experimenter handling the objects.

Participants indicated their judgments of the stable point by lightly grasping the object with the tongs at the appropriate location. Participants never lifted the object. The experimenter measured the error in estimation by noting the angle and the radial distance of the point of contact (in millimeters) in the polar coordinates on the back of the object.

Size was manipulated as a between-subjects variable. Originally, we ran three different experiments with 10 participants in each, resulting in an unbalanced between-subjects manipulation of size (the reason for the irregular sizing of the objects). A given observer judged different size levels across the six shapes. (The quadrilateral was used in only one of the original experiments. Here, we report results from the six remaining shapes, with data from the quadrilateral being used only to determine the axes for measurement in a subsequent experiment.)

Observers viewed all of the objects at three orientations: $0^{\circ}, 135^{\circ}$, and $270^{\circ}$. We used three orientations instead of four, to reduce the total number of trials and to include off-axis orientations in the maximally symmetric objects. The $0^{\circ}$ orientation was determined for the rectangle and the isosceles and equilateral triangles by orienting the longest reflective symmetry axis vertically. In the case of the right triangle, the longest axis running through the center of mass was used. All triangles were oriented at $0^{\circ}$ with the point down. For the parallelogram, we used an axis connecting the center of mass to one of the obtuse angles. For the square, a nondiagonal symmetry axis was used. Each participant saw each of the 6 shapes at 3 orientations 3 times each, for a total of 54 presentations over three blocks of trials. Presentation order within each block was randomized. Each experimental session lasted approximately $1 \mathrm{~h}$.

\section{Results and Discussion}

Measurements in polar coordinates were converted to Cartesian coordinates with the origin at the center of mass. Polar angle of $0^{\circ}$ was designated as the positive $x$ direction. The spatial distribution of the data for each object exhibited an elliptical shape with distinct major and minor axes. The $x$-data coordinates were regressed on the corresponding $y$ coordinates to determine if the major axis in the distribution matched the axis chosen beforehand as the $x$-axis on the object. In the parallelogram (and quadrilateral), the regression line did not match the $x$-axis. For these, the coordinates were rotated to fit the $x$-axis to the data. The angle of rotation was computed from the slope of the linear regression. Subsequent analysis, therefore, was performed with respect to axes that were intrinsic to the data.

We used the means of the $x$ and $y$ data for analysis of the systematic error along the $x$ - and $y$-axes. Standard deviations calculated for each participant across orientation and trials were used to analyze the random error along the $x$ - and $y$-axes. (We computed standard deviations within orientations for trials both across and within subjects, but we found no effect of orientation on random error in either instance.)

The shapes were ordered according to increasing symmetry as follows: The number of axes of reflective symmetry was counted, as was the number of self-congruences within a $360^{\circ}$ rotation within the plane of the figure. These numbers were added for each shape, and the sum was incremented by 1 if the figure exhibited radial symmetry. The totals gave the following order: (quadrilateral [total symmetry $=1]$ ), right triangle (1), isosceles triangle (2), parallelogram (3), rectangle (5), equilateral triangle (6), square (9).

Systematic errors were very small (within $\pm 4 \mathrm{~mm}$ ), and increased when the major axis of reflective symmetry was vertical. Judgments tended to undershoot the center of mass in that case. Systematic errors were analyzed by performing multiple regressions of total symmetry, orientation, and size, with three two-way and one three-way interaction vectors on the $x$ and then on the $y$ data. Both orientation and size were coded as categorical variables using orthogonal coding. Means computed within each cell across the three trials for each participant were used for this analysis. The analysis was significant for $x$ data $\left[F(7,532)=6.5, r^{2}=.08, p<.001\right]$. Significant main effects were obtained for orientation $(p<.001, \beta=$ -.32 , partial $F=18.3$ ) and for size $(p<.01, \beta=$ -.20 , partial $F=6.8$ ). Total symmetry was only significant as part of the three-way interaction. Overall means for the three size levels only varied within $\pm 1 \mathrm{~mm}$. Systematic errors were affected primarily by orientation. The overall means for the three orientations were $2.01,-.31$, and -.81 , respectively. The consistent tendency was to 
undershoot the center of mass, although less so in the second and third orientations. When the longest axis of reflective symmetry was oriented vertically, mean judgments were approximately $2-3 \mathrm{~mm}$ below the center of mass.

This analysis was also significant when performed on $y$ data $\left[F(7,532)=3.2, r^{2}=.04, p<.002\right]$. However, only the size and size $\times$ orientation interaction were significant, and the means only varied within $\pm 1 \mathrm{~mm}$ of the center of mass.

Random error along the $x$-axis increased with increasing object size but decreased with increasing object symmetry. The pattern was similar but much weaker along the $y$-axis. $x$ and $y$ error levels converged at the square. Random error was analyzed by performing regressions on standard deviations computed for each participant (or size) and shape across trials and orientations. Linear regressions of total symmetry on $x$ standard deviations for each size were all significant $(p<.001)$. Slopes increased from -.38 to -.44 to -.77 for small to large sizes, as did the intercepts, from 6.2 to 6.8 to 9.4 . Slopes were extremely shallow for $y$ standard deviations, and only the linear regression for the largest size objects was signifi- cant $(p<.02)$, with a slope of -.22 and an intercept of 4.1. A multiple regression of total symmetry, size (coded orthogonally), and an interaction vector on all $x$ standard deviations was significant $[F(3,176)=31.3$, $\left.r^{2}=.35, p<.001\right]$. Total symmetry $(p<.001, \beta=$ -.52 , partial $F=72.7)$, size $(p<.001, \beta=-.52$, partial $F=20.2$ ), and the interaction ( $p<.001, \beta=$ .38 , partial $F=11.0$ ), were also all significant. The mean $x$ and $y$ standard deviations for each shape and size were plotted in order of increasing symmetry (Figure 3). No increases in random error occurred with increasing size for the most symmetric shape (the square). When the regressions were performed using indices of reflective symmetry or rotational symmetry, the results were essentially the same as for total symmetry. Use of only radial symmetry accounted for about half of the variance covered by the other symmetry measures.

In summary, the results showed that systematic error was affected by orientation. When the long axis in an object was aligned with gravity, the participants tended to undershoot the center of mass by a larger amount. In contrast, random error was affected by object shape and size: in less symmetric objects, random error increased with

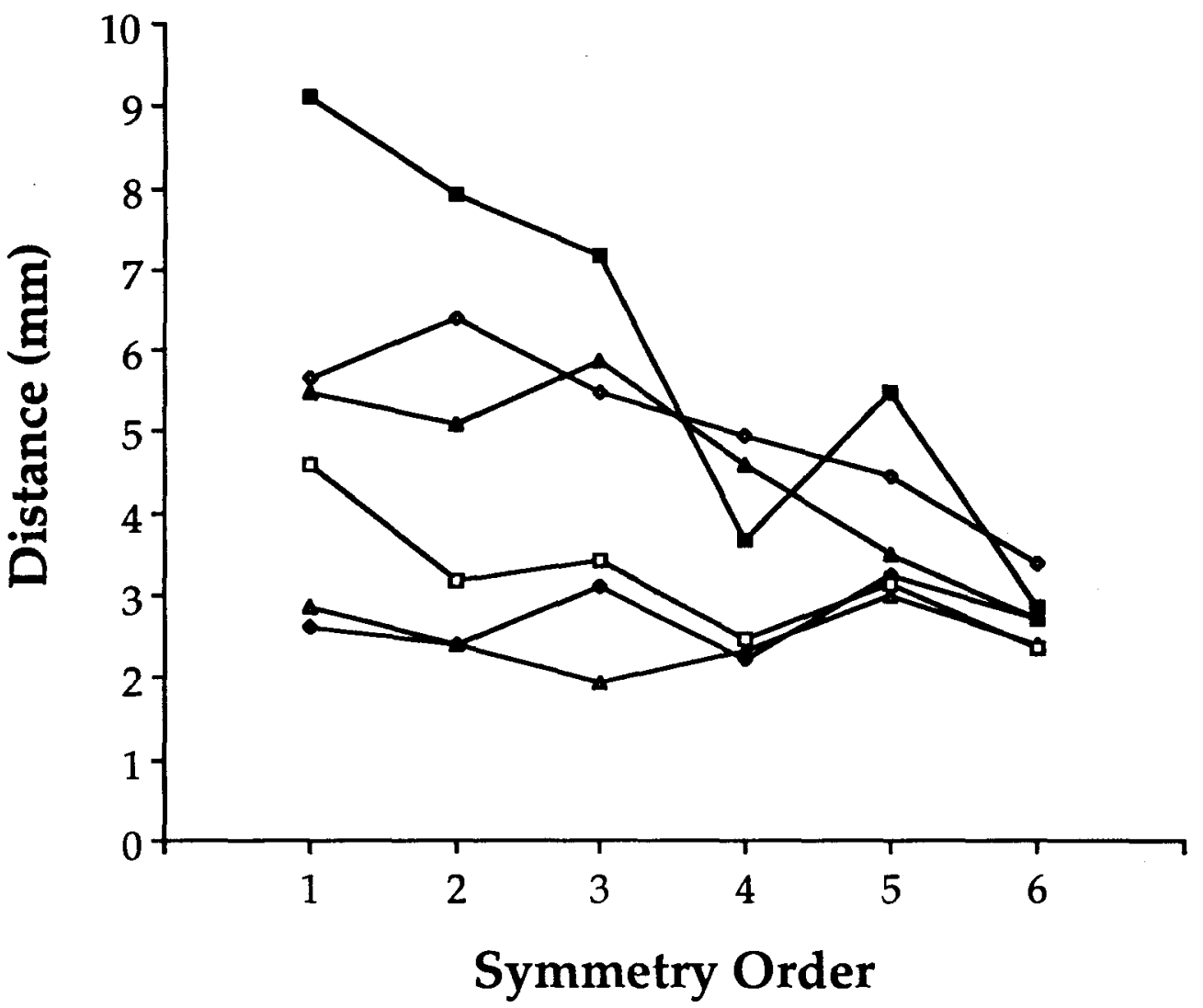

Figure 3. Random errors along the $x$ - and $y$-axes; mean standard deviations for each shape and size are shown, with shapes ordered by total symmetry $x$-axis: large objects $=$ filled squares, medium objects $=$ open diamonds, small objects $=$ filled triangles. $y$-axis: large objects $=$ open squares, medium objects $=$ filled diamonds, small objects $=$ open triangles. 
size, and as the amount of symmetry increased, random error decreased. Random error was greater along the longer $x$-axis than it was along the $y$-axis.

Two of these results, when combined, implied that the observers were well able to recognize and use axes of reflective symmetry occurring in any orientation. First, that random error decreased with an increasing number of reflective symmetry axes implied that the axes were recognized and used; and second, that orientation had no effect on random error implied that the axes were recognized and used in all orientations. Thus, while Rock (1973) may have shown that reflective symmetry axes are recognized less readily in vertical orientations, ultimately they can nevertheless be recognized and used in all orientations.

A number of questions remained unanswered by Experiment 1 . First, what was the contribution of the different types of symmetry to judgment accuracy? Radial symmetry seemed to be a less powerful, but not irrelevant, determinant of judgment accuracy, while the effects of axial reflective symmetry and rotational symmetry could not be determined independently. There was also the question of whether the tendency to undershoot the center of mass more strongly in the first orientation was specific to the particular orientation of the shapes rather than to a vertical orientation of the long $x$-axis: Would undershooting also result at $180^{\circ}$ from this orientation? Finally, related to the effects of orientation, was the question of what determined the axes or orientations intrinsic to the objects that were revealed by the data distributions?

There were also a number of design problems in Experiment 1 . The most obvious was that of the unbalanced and irregular manipulation of size. In addition, use of a between-subjects design was perhaps less sensitive than a completely within-subjects design that might enable us to better differentiate the effects of different types of symmetry. Finally, varying orientation by four increments of $90^{\circ}$ each would have better revealed the effects of orientation with respect to the intrinsic axes found in the data.

\section{EXPERIMENT 2}

All seven shapes shown in Figure 1 were used in three sizes, preserving area across shapes in each size. Objects were presented at four orientations, each separated by $90^{\circ}$ and starting with the $x$-axis vertical, positive end downwards. The axes used were those found in the data of Experiment 1 . The positive $x$-axis (or $0^{\circ}$ polar radius) on the quadrilateral ran from the center of mass to a point about $20^{\circ}$ clockwise from the smallest acute angle. The $x$-axis on the parallelogram connected the midpoints along the short sides. All three factors (shape, size, and orientation) were within subjects.

\section{Method}

Participants. Ten undergraduate students at Indiana University participated in the experiment, and were paid $\$ 4.25$ per hour. All of the participants had normal or corrected-to-normal vision and reported no motor disabilities.
Apparatus. The quadrilateral, shown in Figure 1, was added to the six shapes used in Experiment 1, and was included as a nontriangular asymmetric shape. Three sizes were used in all shapes, with surface areas of 100,200 , and $300 \mathrm{~cm}^{2}$. Seven shapes in three sizes yielded 21 objects in total.

Procedure. The task was the same as in Experiment 1. Each participant judged all 21 objects at 4 orientations 3 times each, for a total of 252 presentations divided into two sessions, each lasting $75 \mathrm{~min}$ and held on consecutive days. Presentation order within each of three blocks of trials was randomized.

\section{Results and Discussion}

Systematic error was analyzed by performing repeated measures analyses of variance (ANOVAs) on the $x$ and $y$ data, with shape, orientation, and size as factors. For the $x$ data, the size and shape factors were not significant, but the orientation factor was $[F(3,27)=11.5, p<$ $.001]$, as was the size $\times$ orientation interaction $[F(12,108)$ $=3.8, p<.001]$. In a simple effects test, sizes were significantly different at Orientations 1 and $4(p<.04)$, while orientation was significant at all levels of size $(p<$ $.001)$. The means are plotted in Figure 4a. Other interactions were significant also, but in each case the means varied only by $\pm 1 \mathrm{~mm}$ and the patterns seemed random.

For the $y$ data, the shape factor was significant $[F(6,54)$ $=5.5, p<.001]$, but all means were within $\pm 1 \mathrm{~mm}$ except for that of the quadrilateral, which was at $1.8 \mathrm{~mm}$. Both orientation $[F(3,27)=9.3, p<.001]$ and size $[F(2,18)=28.8, p<.001]$ were significant. The interaction was not. The means also are plotted in Figure 4a. As can be seen in Figure 4a, the $x$ and $y$ means were affected primarily by orientation. To reveal the pattern of change over orientations for the centroids of the distributions, the overall means were plotted in $x, y$ coordinates (see Figure $4 \mathrm{~b}$; arrows show orientation). The trend was for the centroids to be located below and to the left of the center of mass by about $2-3 \mathrm{~mm}$.

Random errors were analyzed by computing $x$ and $y$ standard deviations across trials within shapes, sizes, and orientations for each participant. A repeated measures ANOVA was performed on $x$ standard deviations, with size, orientation, and shape as factors. Both size $[F(2,18)$ $=37.9, p<.001]$ and shape $[F(6,54)=33.2, p<$ $.001]$ were significant, as was the interaction $[F(12,108)=$ $2.6, p<.005]$. Neither orientation nor any of its interactions were significant. Standard deviations increased with increases in object size for less symmetric objects. As symmetry increased, random error decreased, eventually eliminating the effect of size in the most symmetric objects. In a simple effects test, size was significant $(p<$ .01 ) for all shapes except the rectangle and square, while shape was significant at all size levels $(p<.01)$.

In a repeated measures ANOVA performed on $y$ data, size $[F(2,18)=14.7, p<.001]$ and shape $[F(6,54)=$ $7.9, p<.001]$, were both significant. The interaction was not. In a simple effects test, shape was significant at all size levels $(p<.001)$, but size was significant only for the quadrilateral and equilateral triangle $(p<.05)$. Orientation was significant $[F(3,27)=7.9, p<.003]$. Examination of the means revealed that random error in- 


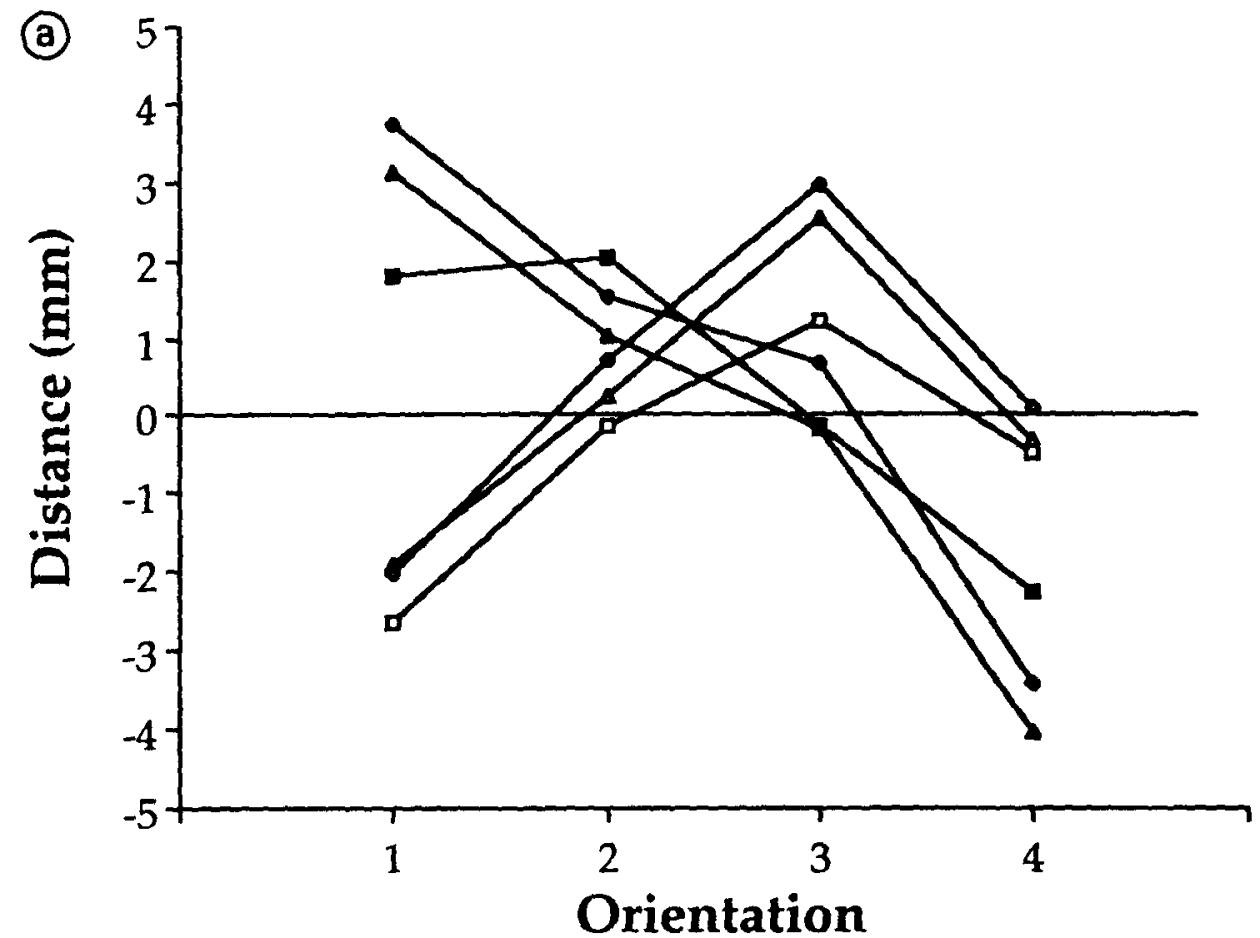

(b)

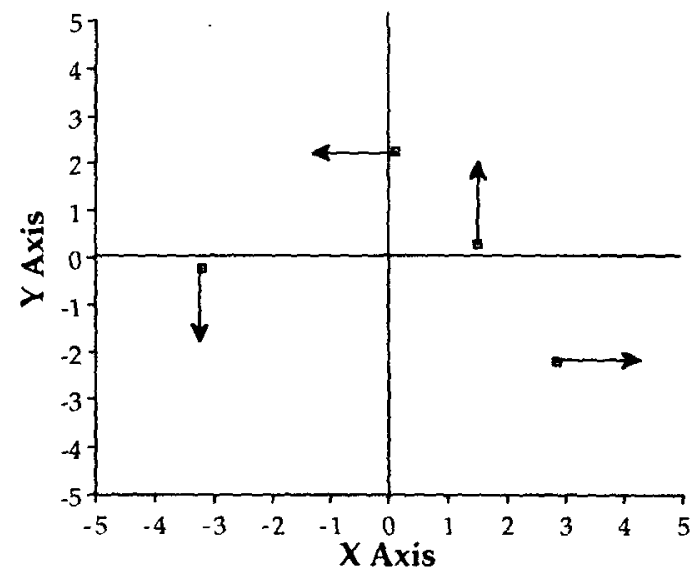

Figure 4. (a) Systematic errors along the $x$ - and $y$-axes for each of the three object sizes, plotted by orientation. $x$-axis: $300 \mathrm{~cm}^{2}=$ filled squares, $200 \mathrm{~cm}^{2}=$ filled diamonds, $100 \mathrm{~cm}^{2} \approx$ filled triangles. $y$-axis: $300 \mathrm{~cm}^{2}=$ open squares, $200 \mathrm{~cm}^{2}=$ open diamonds, $100 \mathrm{~cm}^{2}=$ open triangles. (b) Locations of the centroids of the distributions for each of the four orientations. Arrows indicate the downward direction for each orientation,

creased, especially in more symmetric objects, when the $y$-axis was vertical. The same trend occurred in the $x$ data, although there it failed to reach statistical significance. This can be seen in Figure 5, where vertical versus horizontal mean standard deviations for $x$ or $y$ are plotted separately for each size. Means for the most symmetric objects (rectangle, equilateral triangle, and square) at vertical orientations of both the $x$ - and $y$-axis were greater than those at horizontal orientations. There was a trend in this direction for other shapes as well.

Apparent in Figure 5 was a knee in both $x$ and $y$ judgment curves at Object 6, the equilateral triangle. In Ex- periment 1 , this had appeared only in the $y$ data, and in the $x$ data for the largest size. This knee placed the data for the equilateral triangle closer to those for the isosceles and right triangles and the quadrilateral. The result was an apparent split in the data depending on the presence or absence of radial symmetry.

Except for the square, in which the lengths of the $x$ and $y$-axes were equivalent, $y$ standard deviations were always smaller than $x$ standard deviations. Mean $x$ and $y$ standard deviations were closer also for the quadrilateral, in which the difference in axis lengths was smaller. These observations taken together with the fact that mean stan- 

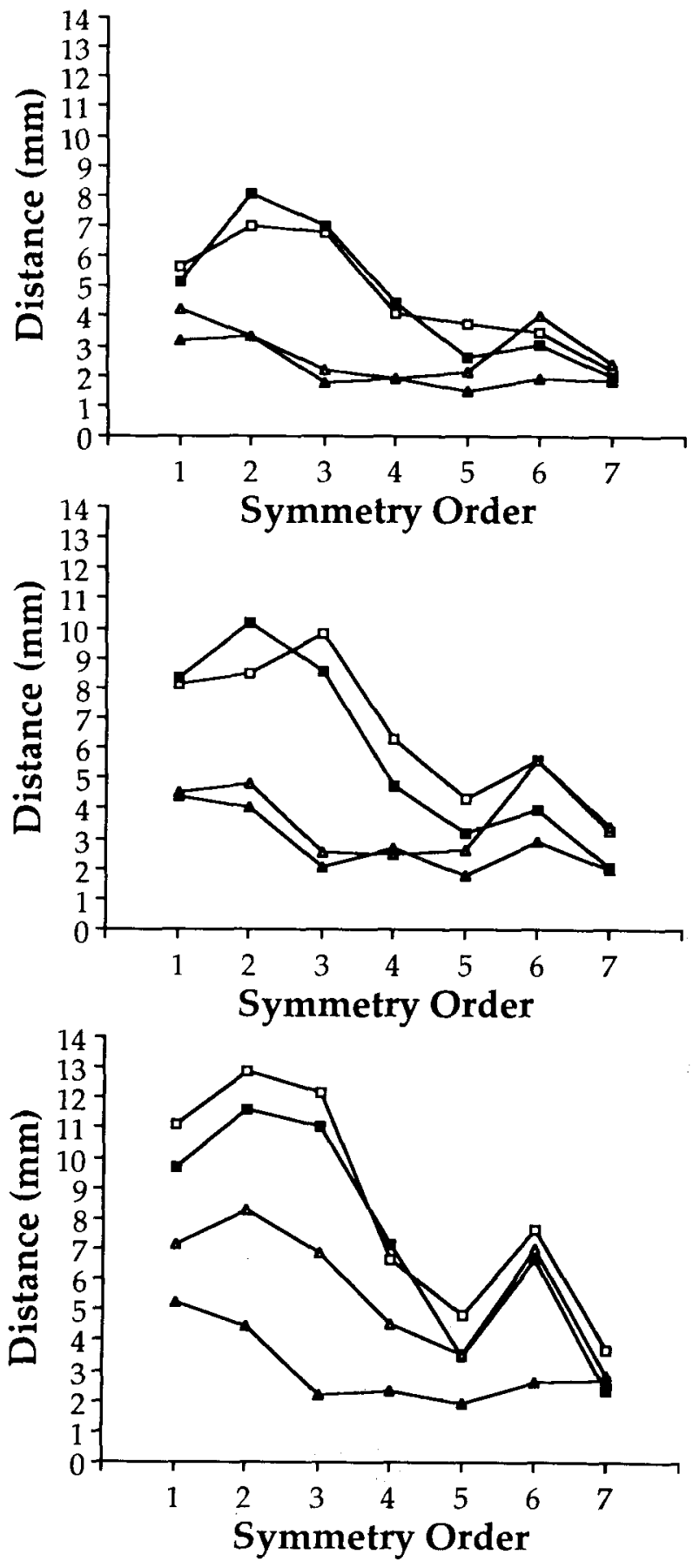

Figure 5. Random errors along $x$ - and $y$-axes for each shape (top $=$ $100 \mathrm{~cm}^{2}$, middle $=200 \mathrm{~cm}^{2}$, bottom $=300 \mathrm{~cm}^{2}$ ). The respective axes vertical or horizontal are plotted separately for each size, and shapes are ordered by total symmetry. Axis vertical = open symbols, axis horizontal $=$ closed symbols; $x$-axis $=$ squares, $y$-axis $=$ triangles.

dard deviations generally increased with object size, as apparent in Figure 5, implied that standard deviations varied generally with axis length. We computed $x$ and $y$ standard deviations for vertical and horizontal orientations of each shape twice - that is, both within and across participants. In both cases, we plotted $x$ - and $y$-axis lengths against $x$ and $y$ standard deviations, respectively. Two separate trends were apparent in scatterplots, and corresponded to shapes with or without radial symmetry. Using the $x$ standard deviations computed for each participant, we performed a multiple regression on $x$ standard deviations with vectors for $x$-axis length, radial symmetry, and the interaction. The regression was significant $[F(3,416)$ $\left.=111.0, r^{2}=.44, p<.001\right]$, as was $x$-axis length $(p<$ $.001, \beta=.54$, partial $F=191.9$ ). Radial symmetry was not significant $(\beta=.15)$, but the interaction was significant $(p<.001, \beta=-.49$, partial $F=24.9)$. The result was the same when the analysis was performed on $x$ standard deviations computed across participants, but the $r^{2}$ was doubled to .84 . When the analysis was performed on $y$ standard deviations within participants, it was significant $\left[F(3,416)=41.8, r^{2}=.23, p<.001\right]$, as was $y$-axis length $(p<.001, \beta=.34$, partial $F=22.0)$ and the interaction $(p<.002, \beta=-.44$, partial $F=$ 4.7). Radial symmetry was not significant $(\beta=.18)$. When this was performed on $y$ standard deviations computed across participants, the $r^{2}$ was doubled to .45 . Including a vector coding for vertical versus horizontal axis orientation improved the $r^{2}$ for the $y$ data but not for the $x$. The trends are shown in Figure 6, where mean standard deviations for each object are plotted against axis lengths with linear fits for data with and without radial symmetry at each of the two orientations.

Random error increased with axis length more rapidly in objects without than in objects with radial symmetry, and increased somewhat more rapidly for vertical than horizontal axis orientations. Similar results were obtained when we performed this analysis on the data from Experiment 1 .

We found that radial symmetry together with axis lengths accounted for a large proportion of the random errors. However, the existence of axes of reflective symmetry was also relevant, as perhaps was the amount of rotational symmetry. We performed separate linear regressions of axis lengths on $x$ and $y$ standard deviations for each object and axis orientation (vertical or horizontal). The slopes of these regressions are shown in Figure 7. Here again, the difference between shapes with and without radial symmetry was apparent. Random errors increased more rapidly with size in shapes without radial symmetry. However, variations depending on the presence or absence of axes of reflective symmetry were also apparent. Although $x$ slopes were comparable in the right and isosceles triangles, the $y$ slope decreased with the presence of the reflective symmetry axis in the isosceles triangle. The same was true of the parallelogram versus the rectangle and square. These differences also appear in the means in Figure 5. Clearly, the existence of axial reflective symmetry also reduced random errors. We could not determine on the basis of these results whether rotational symmetry might contribute to the accuracy of judgments 

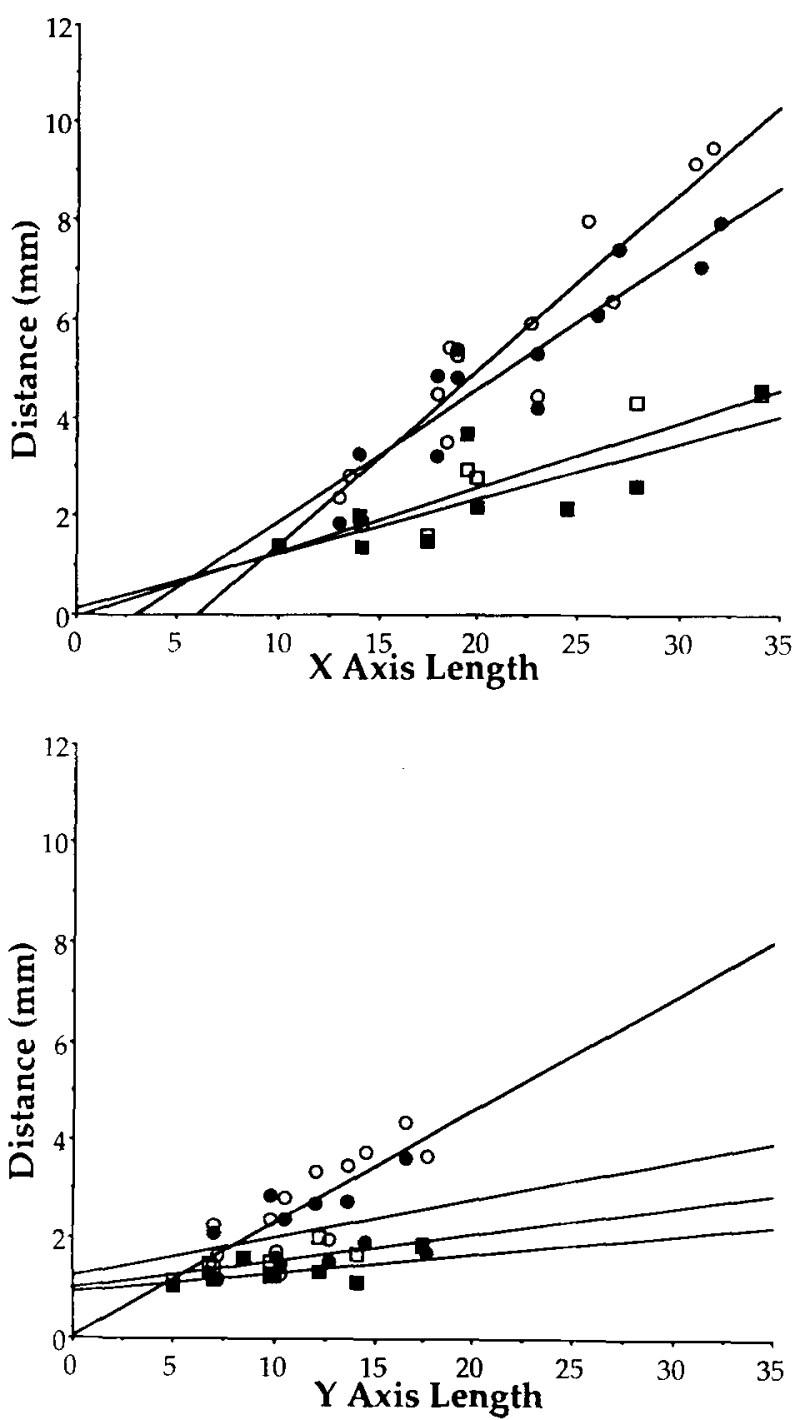

Figure 6. Mean standard deviations for each shape and size and axis orientation (vertical vs. horizontal), plotted against axis length separately for $x$-and $y$-axes. Least squares best fit lines are plotted for shapes with (squares) and without (circles) radial symmetry for each orientation. Vertical orientation of the axis $=$ open symbols, horizontal orientation of the axis $=$ filled symbols.

beyond that allowed by radial symmetry and independent of axial reflective symmetry.

\section{GENERAL DISCUSSION}

Many questions still remained unanswered, but the most pressing concerned the existence of intrinsic object axes in the data and the effects of orientation on the distribution of errors along these axes. We turned to the underlying dynamics for an account in both cases. Regarding the first question, our empirically determined axes aligned rather well with the principal moments of inertia. Having used the first moment or the center of mass to establish the origin of a coordinate system, the next logical step would be to use the second moments or principal moments of inertia to determine the axes themselves. As to the latter question, which we consider first in the following discussion, symmetry eliminated explanation via shape variations, leaving the dynamically determined repercussions of error as the only basis for understanding.

\section{Orientation to Gravity}

We have described this task in terms of center of mass perception. To interpret these results in terms of the perception of either a dynamic or an affordance property might seem inappropriate; after all, we had reduced our object variations to variations in planar shapes. Perhaps our participants were only judging centroids of areas as such-that is, as strictly geometric properties with no dynamic content whatsoever. The problem with such an account, however, is that we did not ask our participants to judge centers of area; we asked them, rather, to judge a dynamically determined dispositional property of the objects-that is, to judge where the objects should be grasped to produce neutrally stable equilibrium in a precision grasp. The task was inherently dynamical. Furthermore, the participants found the task as explained to them to be intuitively clear, and they had no difficulty in understanding what was required of them.

The greatest difficulty for a nondynamical account, however, is in handling variations in judgments corresponding to variations in object orientations. Changes in
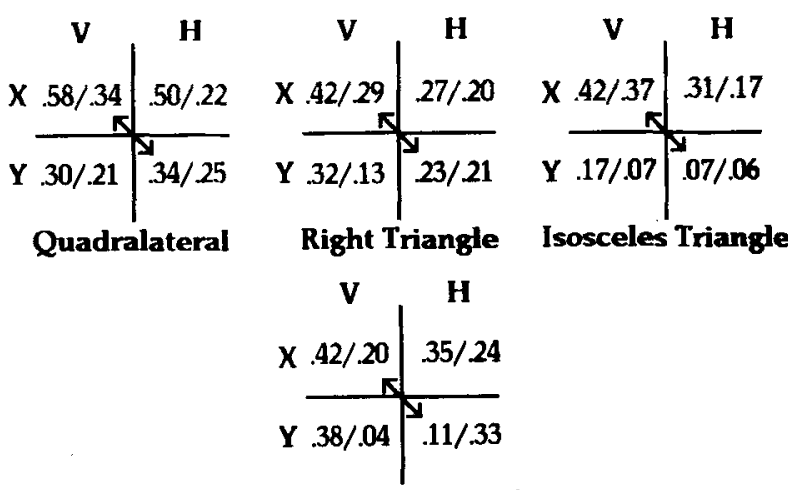

Equilateral Triangle

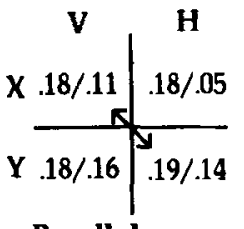

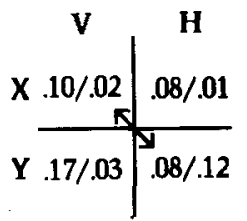

Rectangle

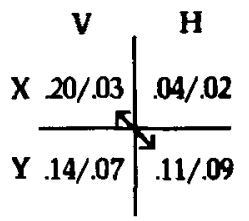

Square
Figure 7. Slopes of linear regressions performed for each shape and axis orientation (vertical vs. horizontal) regressing axis length on standard deviations. The first number in each cell is the slope for the regression performed on standard deviations computed across participants, while the second is for that performed on standard deviations computed within participants. The arrows are reminders that $x$ was vertical while $y$ was horizontal (and vice versa). The top four shapes lack radial symmetry, while the bottom three possess it. 
judgments reflected the gravitational direction. This certainly implied dynamic content. Systematic errors tended to fall below the center of mass. Initially, this tendency was surprising: we had expected to see overshooting in the direction of stable equilibrium rather than undershooting. However, the observed tendency also produced a stable grasp. Because participants were grasping the objects from the right, a systematic tendency to grasp below and to the left of the center of mass would result in a tendency for the object to rotate into the grasp so as to contact and lean against the hand. This also would yield a somewhat better measure of control over the object's trajectory.

We found an orientation effect in random error as well. In the objects with the largest number of reflective symmetry axes, random errors along an axis were greater when it was aligned with gravity. An interesting aspect of this effect was that it occurred strongly with the square; because of its $90^{\circ}$ rotational symmetry period, $90^{\circ}$ changes in orientation of the square were not accompanied by shape-related changes. Generally, the orientation effect on random error seems to have emerged as the objects became more symmetric and shape variations became less of a factor. The effect is illustrated in Figures $8 \mathrm{a}$ and $8 \mathrm{~b}$, respectively, for the equilateral triangle and square, which were the most symmetric objects-one with and one without radial symmetry. The contour plots represent the spatial distribution of judgment frequencies across object surfaces. They reveal the tendency of the distributions to align with gravity in each of the four orientations.

Why should these distributions be constrained in the direction perpendicular to gravity while spreading in the direction of gravity? What was the difference between misjudging above or below the center of mass as opposed to misjudging off to the side? Dynamical analysis revealed the functional repercussions of misjudging the center of mass by distances in different directions. The angular acceleration about the opposition axis (that is, about the points of contact) increases with increasing distance from the center of mass, depending on the direction with respect to gravity. The analysis is shown in Figure 9. The object acts as a physical pendulum, and this determined the equation of motion. We used the parallel axis theorem to compute the moment of inertia around the opposition axisthat is, the inertia was computed as the inertia around the center of mass added to the product of the object mass and the square of the distance from the center of mass. The inertia about the center of mass was computed as a function of two shape-specific constants and the squares of object dimensions. When these were substituted into the original expression, the masses canceled, meaning that the rotational acceleration was strictly a function of the geometry of the object and gravity. $\theta$ and $r$ can be thought of as polar coordinates on the object surface with the origin at the center of mass. For each object of a given shape, the rotational acceleration was computed for each potential contact point on the object surface using this function as a single-valued function (the rotational acceleration) in two variables $(\theta$ and $r)$. All objects yielded acceleration surfaces with the same basic shape: two hills surrounding a vertically oriented valley containing the center of mass. The valley always aligned with gravity. Only the relative height of the hills and the steepness of their slopes varied with shape. The basic form of the acceleration surface is shown in Figure 10 from two perspectives. We suggest that the elliptical distributions in the data tend, all else being equal, to lie along the valley in this plot.

Note that this plot is the product of a very local analysis in time. As a resuit, the plot does not reflect the asymmetry in the stability properties of points located above versus below the center of mass. The surface represents the accelerations generated instantaneously at the given location. This is relevant when a complete lack of rotation is desired (as opposed to rotations that take one into desirable configurations). The problem, of course, is that corrections or responses to rotations can only be provided in some finite time. The greater the rotational acceleration, the greater the amount of undesired rotation within a given response time (or alternatively, for a given minimum amount of rotation, the smaller the time in which to respond and the greater the required vigilance). To minimize amplitude of rotation in slips, the optimal strategy would be to hone as closely as possible to a vertical line extending though the center of mass. This is what the participants appear to have done when best able to recognize a vertical passing though the center of mass.

\section{Inertial Frames of Reference}

We have noted that in objects with maximum symmetry, the elliptical distributions tended to align with gravity. In all cases, however, the axes of the elliptical distributions aligned within the object geometry. Furthermore, the major axis of the ellipse tended to align with object shape, especially when there were fewer than two axes of reflective symmetry. How might we account for these orientations? The major axes of the distributions did not consistently align with the longest axis passing though the center of mass. In objects with axes of reflective symmetry, the axes of the distributions aligned with the symmetry axes. But what of the asymmetric objects or the objects with only radial symmetry? This problem has been considered at length elsewhere in the context of object or shape recognition.

In the study of shape recognition, a controversy has developed concerning the frame of reference used to establish orientation for purposes of recognition. The alternatives have included a retinal (Corballis \& Roldan, 1975; Sutherland, 1968), a gravitational (Hock \& Tromley, 1978; Rock, 1973, 1983), and an intrinsic (based on properties of object shape) frame of reference (Hinton, 1981a, 1981b, 1981c; Hock \& Tromley, 1978; Marr, 1982; Wiser, 1981). The controversy has been conditioned by the assumption that perception proceeds by constructing descriptions of objects. Recognition has been portrayed as a matter of matching current descriptions with stored 
(a)
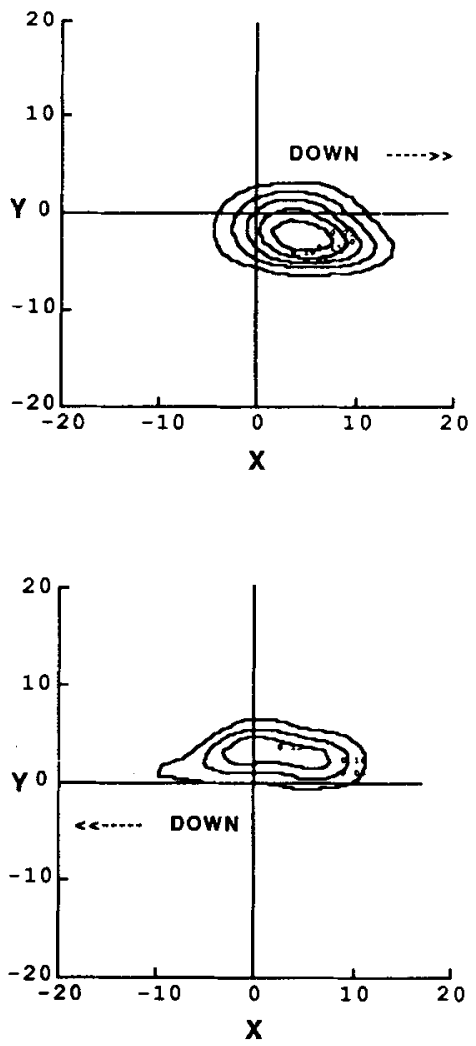

(b)
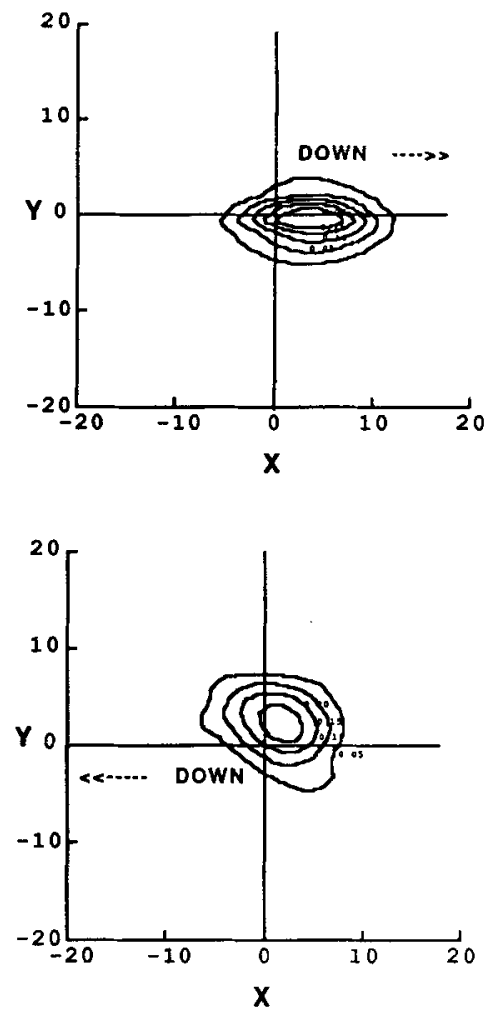
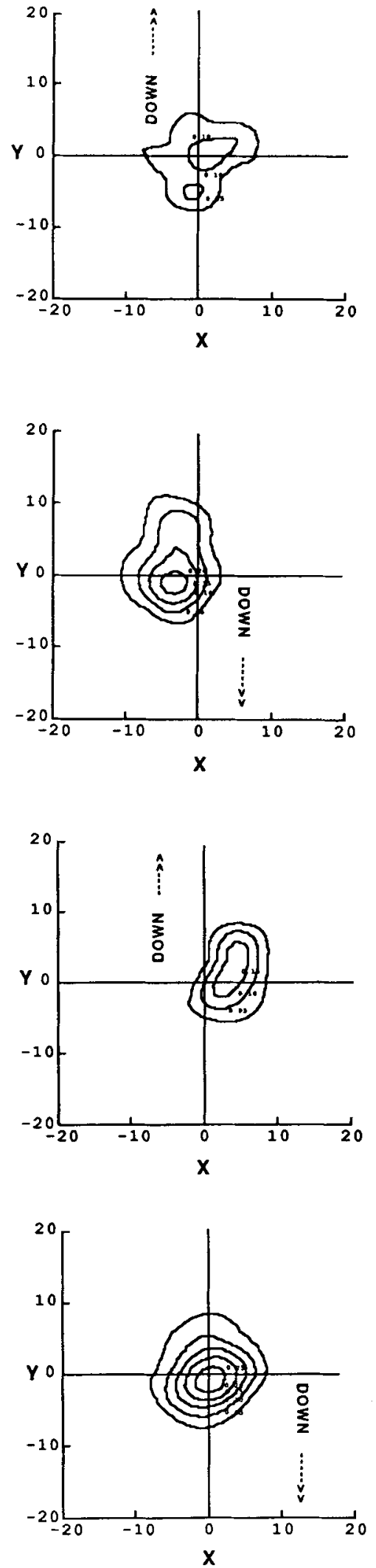

Figure 8. Contour plots of spatial frequency distributions plotted separately for each orientation, for equilateral triangles (a) and squares (b). Arrows indicate the downward direction for each orientation. 


\section{Calculation of Rotational Acceleration}

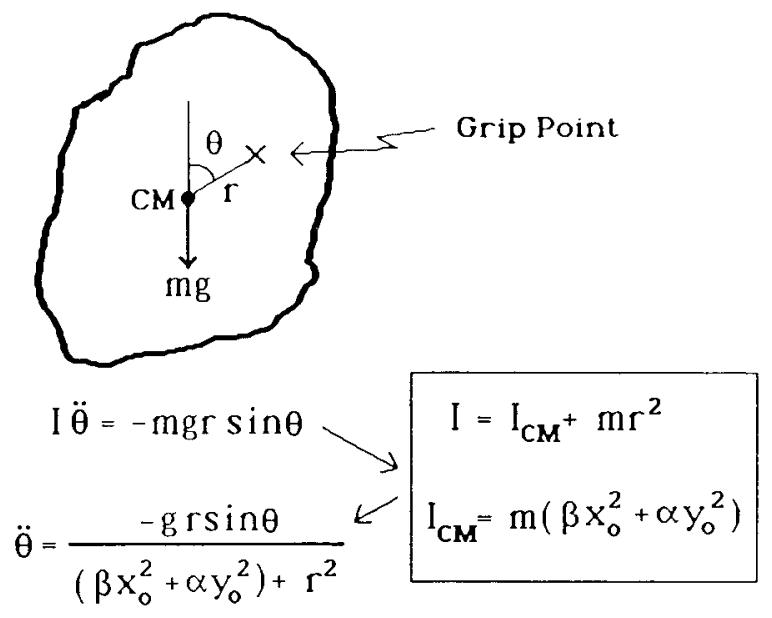

Figure 9. Calculation of the instantaneous rotational acceleration about the opposition axis.

descriptions. The extrinsic geometries typically used to model this process require a set of coordinates to describe and to match shapes. ${ }^{4} \mathrm{~A}$ frame of reference is used to determine the orientation of the coordinate axes. A frame intrinsic to object shape has been advocated in the interests of parsimony. The idea has been to reduce the number of stored representations used to recognize an object. Although shape recognition seems relatively insensitive to changes in size and location (Corcoran, 1971; Deutsch, 1955; Sutherland, 1960, 1968; Sutherland \& Carr, 1963), it can be perturbed by changes in orientation ${ }^{5}$ (Attneave \& Olson, 1967; Attneave \& Reid, 1968; Corballis, Anuza, \& Blake, 1978; Corballis \& Roldan, 1975; Corballis, Zbrodoff, \& Roldan, 1976; Hock \& Tromley, 1978; Quinlan, 1991; Rock, 1973, 1983; Rock, DiVita, \& Barbeito, 1981; Rock \& Leaman, 1963). This sensitivity has been used to argue for gravitational rather than intrinsic frames of reference.

A large proportion of studies on shape recognition have focused on the recognition of two-dimensional (2-D) planar shapes, with the suggestion that their results might be generalized to the perception of 3-D object shape, assuming that the latter is mediated by momentary, static, 2-Dprojected shape (Quinlan, 1991). The problem with this idea is that it confuses the shape of an object or of a figure drawn on a surface with optical information about shape. The optical information about object shape or drawn shape is never a copy of the shape. Shape in optical pattern is transformed by the nonlinear (that is, polarprojective) mapping to the point of observation. The momentary optical pattern depends on the momentary perspective. RT studies have attempted to control optical flow resulting from continuous change in perspective by using tachistoscopic exposures. This method has been used in studies that have produced results used to argue in favor of a retinal frame of reference (Corballis et al., 1978; Cor- ballis \& Roldan, 1975; Corballis et al., 1976; Quinlan, 1991). The results produced using this method are of questionable generality because the mapping from object shape (or surface structure) to optical pattern has been restricted in a way that is entirely unrepresentative. Free variation in the perspective mapping is an unavoidable facet of the visual perception of shape. A more promising approach, recognizing that the observer is never motionless, is to study the regularities in the optical flow patterns that are generated over perspective transformations (Koenderink, 1975; Koenderink, 1986; Koenderink, 1990; Koenderink \& van Doorn, 1978; Nakayama, 1985; Thompson, 1989; Thompson, Mutch, \& Berzins, 1984, 1985; Waxman \& Ullman, 1985). We must emphasize that we have not pursued this aspect of center of mass perception here. Our results (and a large proportion of results in the shape-perception literature) concern variations in object shape and orientation. We emphasize that these shapes should not be confused with optical information about shape.

If we interpret the debate in the shape-perception literature in a manner that is consistent with the distinction
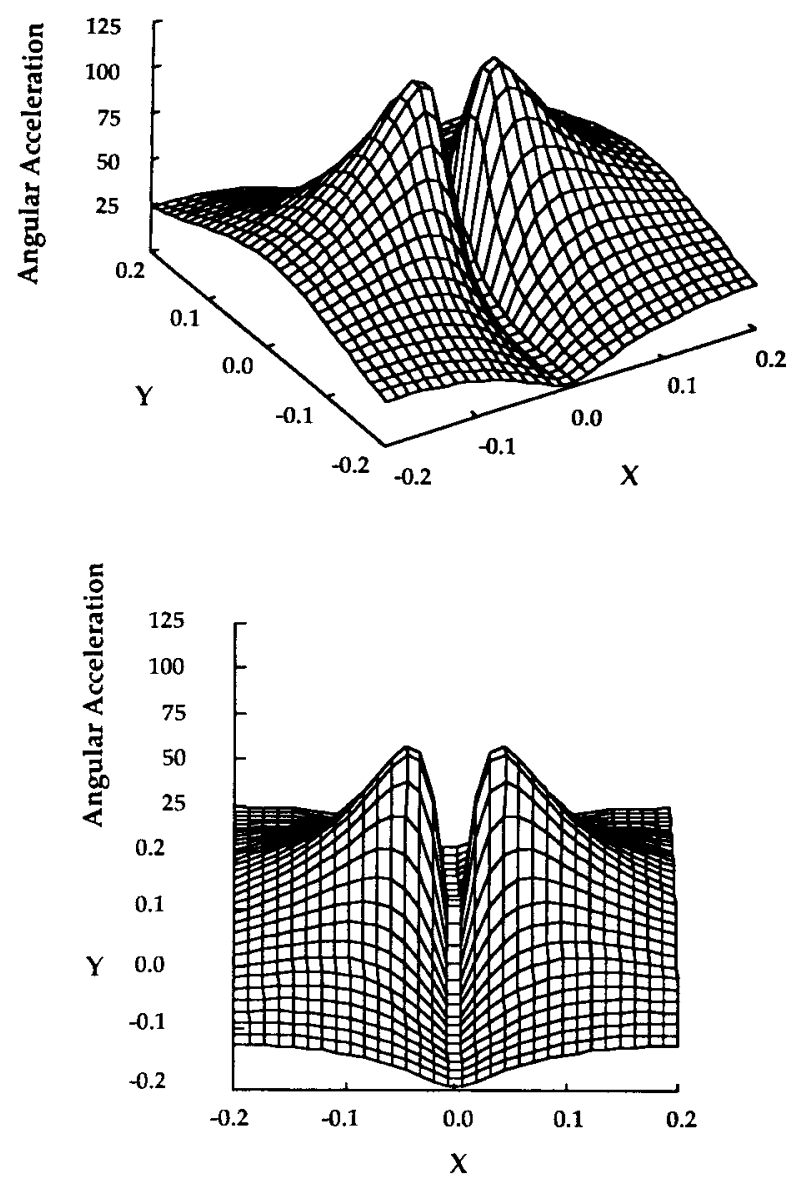

Figure 10. An illustrative rotational acceleration surface shown from two perspectives. $x$ and $y$ distances are in meters, while angular acceleration is in radians/second. 
between optical flow and object shape, then the controversy has been about fixing coordinates in an object. The strategy for fixing the origin has been to use the center of mass (or the center of area) ${ }^{6}$ (Ballard \& Brown, 1982; Horn, 1986; Kanade \& Kender, 1983; Marr, 1982; Wechsler, 1990). This strategy has been common to almost all approaches despite disagreement over the means of establishing coordinate orientation. In machine or computational vision, orientation of coordinates has been achieved exclusively via object-centered frames of reference. Two largely overlapping approaches can be distinguished. In the more general computational approach, axes of reflective symmetry and direction of elongation have been hypothesized as object properties used to fix coordinates (Horn, 1986; Kanade \& Kender, 1983; Marr, 1982; Quinlan, 1991; Wiser, 1981). In the absence of reflective symmetry, the longest axis passing through the center of mass in an object or figure is used. (The center of mass is used because the longest axis otherwise corresponds to an edge in some figures, such as triangles and rhombuses.)

An alternative approach commonly employed in robotics is to use the second moments, the principal axes of inertia (Horn, 1986). This is a logical extension of having used the first moment or center of mass to establish the origin of the coordinates. The principal axes of inertia are orthogonal axes fixed at the center of mass. As such, they are Cartesian coordinate axes that are intrinsic to a given shape. They represent axes of rotation at which inertial resistance is maximal at one axis while minimal at the other. They also correspond to axes around which rotation is smoothest and most stable. Although the larger of the principal axes may pass near the longest axis through the center of mass, most often it is not the same as the longest axis. The two approaches to establishing intrinsic object coordinates therefore differ, but only in this respect; otherwise, the principal moments correspond to axes of reflective symmetry when the latter exist.

Use of the inertial axes is certainly a more elegant approach than using either symmetry or the longest axis, the choice between which is problematic for objects in which symmetry is closely approximated. Objects are precisely symmetric only on rare occasion. How much must an object deviate from symmetry before the longest axis is used in preference to an (approximate) symmetry axis? The difference may be significant. For instance, in a rectangle, the longest reflective symmetry axis connects the midpoints of the shorter sides, while the longest axis through the center of mass runs corner to corner. As the rectangle is perturbed gradually into a parallelogram, at what point would the coordinate axis jump to the corner? The inertial axis moves continuously away from the midpoint of the sides toward the (acute-angled) corners as a rectangle is transformed into a parallelogram with angles that gradually move farther from $90^{\circ}$. The location of the inertial axes is well defined at every configuration of the figure.

Using the inertial axes is preferable also because they are directly relevant to the control of object manipula- tion. (Naturally, roboticists would appreciate this advantage.) A growing number of studies have shown that people are very sensitive to the inertial properties of objects that they either manipulate (Bingham et al., 1989; Jones, 1986; Solomon \& Turvey, 1988; Solomon, Turvey, \& Burton, 1989a, 1989b; Turvey, Burton, Pagano, Solomon, \& Runeson, 1992) or might manipulate (Bingham, 1987b; Runeson \& Frykholm, 1981, 1983). In particular, Solomon et al. (1989a, 1989b) have demonstrated a sensitivity to the inertial moments.

The alignment of the distributions in our center of mass judgments supported the inertial-axes approach to object coordinates. First, as shown in Figure 11, the axes in the distributions aligned with axes of reflective symmetry. In these cases, the longest symmetry axis was not always the longest axis through the center of mass-it was in the triangles, but was not in the squares and rectangles. In the elongated objects, the major axis of the ellipse tended to run in the direction of elongation. The exceptions, of course, were the objects with multiple axes of reflective symmetry, one of which lay parallel to gravity; in such cases, the ellipse also tended to align with gravity. We did not record an effect of orientation on random errors in Experiment 1, in which we aligned reflective symmetry axes with gravity only at one orientation.

We used the orientations in the distributions recorded in Experiment 1 to determine the axes used for orientation and analysis in Experiment 2. The distributions in
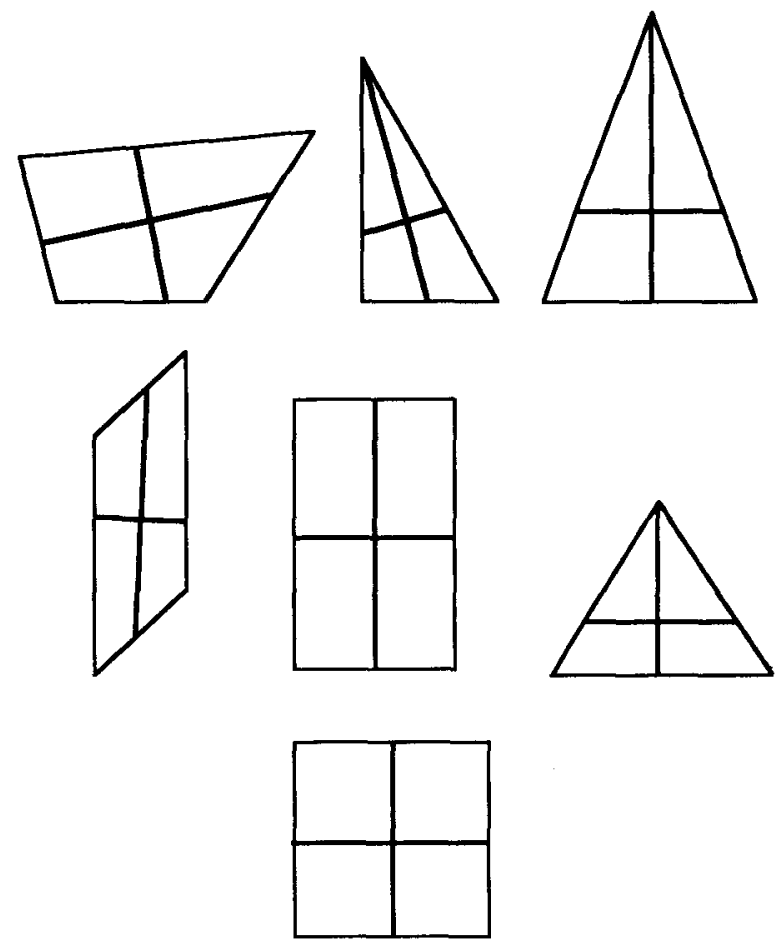

Figure 11. Locations of the $x$ and $y$ principal moments of inertia compared with the axes apparent in the data distributions for each of the shapes (the two coincided). 
Experiment 2 aligned with the axes determined in Experiment 1 with one exception. Without knowing or thinking about the locations of the inertial axes, we had allowed ourselves in Experiment 1 to be influenced by the approximate symmetries of the parallelogram. The choice seemed to be whether to lay the $x$-axis between the two acute corners or between the midpoints of the short sides, since the major axis in the data lay $5.33^{\circ}$ toward the acute corners from an axis between the midpoints of the short sides. We interpreted the $5.33^{\circ}$ as measurement error and placed the axis for Experiment 2 between the midpoints of the short sides. As it happens, the axis of the larger of the principal moments of inertia lies almost exactly where the Experiment 1 data were-about $5^{\circ}$ above the axis that we used in Experiment 2. Likewise, the orientation of the data distributions for the parallelograms in Experiment 2 was $7.5^{\circ}$ toward the acute corners, as shown in Figure 11.

We determined these orientations by regressing $x$ on $y$ data for each of the four object orientations, collapsing across sizes, and then computing the mean slope across the four orientations. Because the major axis of the elliptical distributions lay along the $x$-axis in all cases except that of the parallelogram, these regressions were nonsignificant (with slopes near 0 ) on at least three occasions out of four for each object. For the parallelogram, three of the regressions were significant $(p<.001)$ and, as mentioned, the mean slope was $7.5^{\circ}$ with a standard deviation of $5.8^{\circ}$. The longest axis through the center of mass in a parallelogram is the axis running between the acute corners. This was about $14^{\circ}$ from the axis between the midpoints of the short sides and $6.5^{\circ}$ (that is, more than a standard deviation) away from the mean orientation of the data distribution. The major axis in the data distributions for the quadrilateral did not coincide with the longest axis in the objects either, although, once again, it was about $2.5^{\circ}$ from the inertial axis in the direction of the longest axis.

Taken by themselves, the results for the parallelogram and the quadrilateral are perhaps not absolutely conclusive evidence that axes reflected in performance correspond to inertial axes. However, determination by inertial axes is certainly the more parsimonious approach when such evidence is coupled with the alignment of data with symmetry axes when they exist. The frequent noncoincidence of long axes and reflective symmetry axes places the two at odds in a way that would require complex criteria for their respective application. Furthermore, inertial axes are of greater potential use and we have other evidence that these properties are used in object manipulation.

In any case, our observers were clearly skilled in determining the location of the center of mass in these objects; errors rarely exceeded a finger's width in distance from the actual center of mass. Observers were more accurate with objects that exhibited radial symmetry, and also appeared to use reflective symmetry. Whether rotational symmetry is also used remains unclear, and the issue of what is done in the absence of symmetry is also still to be determined, although a sensitivity to inertial moments would constrain the possibilities.

\section{REFERENCES}

Attneave, F., \& Olson, R. K. (1967). Discriminability of stimuli varying in physical and retinal orientations. Journal of Experimental Psychology, 74, 149-157.

Attneave, F., \& ReID, K. W. (1968). Voluntary control of frame of reference and slope equivalence under head rotation. Journal of Experimental Psychology, 78, $153-159$.

Ballard, D. H., \& Brown, C. M. (1982). Computer vision. Englewood Cliffs, NJ: Prentice-Hall.

BECKER, R. A. (1954). Introduction to theoretical mechanics. New York: McGraw-Hill.

Bingham, G. P. (1987a). Dynamical systems and event perception: A working paper. Parts I-III. Perceiving-Acting Workshop Review, 2, 4-14.

Bingham, G. P. (1987b). Kinematic form and scaling: Further investigations and the visual perception of lifted weight. Joumal of Experimental Psychology: Human Perception \& Performance, 13, 155-177.

Bingham, G. P. (in press). Dynamics and the problem of visual event recognition. In R. Port \& T. van Gelder (Eds.), Mind as motion: Dynamics, behavior \& cognition. Cambridge, MA: MIT Press.

Bingham, G. P., \& Muchisky, M. M. (1990, May). Center of mass perception. Paper presented at the meeting of the International Society for Ecological Psychology at the Beckman Institute, University of Illinois, Urbana.

Bingham, G. P., \& Muchisky, M. M. (1991a, March). Center of mass perception for the visual guidance of grasping. Paper presented at the Conference on Human Error sponsored by the Institute for the Study of Human Capabilities at Indiana University.

Bingham, G. P., \& Muchisky, M. M. (1991b, August). Center of mass perception for the visual guidance of grasping: A GSD problem. Paper presented at the 6th International Conference on Event Perception and Action, Amsterdam.

Bingham, G. P., \& Muchisky, M. M. (in press). “Center of mass perception' ': Affordances as dispositions determined by dynamics. In J. Flach, P. Hancock, \& K. Viscente (Eds.), The ecology of humanmachine interactions. Hillsdale, NJ: Erlbaum.

Bingham, G. P., Rosenblum, L. D., \& Schmidt, R. C. (1993). Dynamics and the orientation of kinematic forms in visual event recognition. Manuscript submitted for publication.

Bingham, G. P., Schmidt, R. C., \& Rosenblum, L. D. (1989). Hefting for a maximum distance throw: A smart perceptual mechanism. Journal of Experimental Psychology: Human Perception \& Performance, 15, 507-528.

Bingham, G. P., Schmidt, R. C., Turvey, M. T., \& Rosenblum, L. D. (1991). Task-dynamics and resource dynamics in the assembly of a coordinated rhythmic activity. Journal of Experimental Psychology: Human Perception \& Performance, 17, 359-381.

Corballis, M. C., Anuza, T., \& Blake, L. (1978). Tachistoscopic perception under head tilt. Perception \& Psychophysics, 24, 274-284.

Corballis, M. C., \& Roldan, C. E. (1975). Detection of symmetry as a function of angular orientation. Journal of Experimental Psychology: Human Perception \& Performance, 1, 221-230.

Corballis, M. C., Zbrodoff, J., \& Roldan, C. E. (1976). What's up in mental rotation? Perception \& Psychophysics, 19, 525-530.

Corcoran, D. W. J. (1971). Pattern recognition. Middlesex, U.K.: Harmondsworth.

DEUTSCH, J. A. (1955). A theory of shape recognition. British Journal of Psychology, 46, 30-37.

FEARING, R. S. (1983). Touch processing for determining a stable grasp. Unpublished doctoral dissertation, MIT, Cambridge, MA.

Grünbaum, B., \& ShePHARD, G. C. (1989). Tilings and patterms: An introduction. New York: W. H. Freeman.

Hinton, G. E. (1981a). A parallel computation that assigns canonical object-based frames of reference. In Proceedings of the 7th International Joint Conference on Artificial Intelligence (pp. 683-685). Los Altos, CA. 
Hinton, G. E. (1981b). The role of spatial working memory in shape perception. In Proceedings of the 3rd Cognitive Science Conference (pp. 56-60). San Mateo, CA: Morgan Kaufman

HiNTON, G. E. (1981c). Shape representation in parallel systems. In Proceedings of the 7th International Joint Conference on Artificial Intelligence (pp. 1088-1096). Los Altos, CA:

HoCK, H. S., \& Tromley, C. L. (1978). Mental rotation and perceptual uprightness. Perception \& Psychophysics, 24, 529-533.

HorN, B. K. P. (1986). Robot vision. Cambridge, MA: MIT Press. Iberall, T., Bingham, G. P., \& ARbiB, M. A. (1986). Opposition space as a structuring concept for the analysis of skilled hand movements. In H. Heuer \& C. Fromm (Eds.), Generation and modulation of action patterns (pp. 158-173). Berlin: Springer-Verlag.

JEANNEROD, M. (1984). The timing of natural prehension movement. Journal of Motor Behavior, 26, 235-254.

JEANNEROD, M. (1988). The neural and behavioral organization of goaldirected movements. Oxford: Oxford University Press.

JoNes, L. A. (1986). Perception of force and weight: Theory and research. Psychological Bulletin, 100, 29-42.

KANADE, T., \& KENDER, J. R. (1983). Mapping image properties in shape constraints: Skewed symmetry, afine-transformable patterns, and the shape-from-texture paradigm. In J. Beck, B. Hope, \& A. Rosenfeld (Eds.), Human and machine vision (pp. 237-257). New York: Academic Press.

KoENDERINK, J. J. (1975). Invariant properties of the motion parallax field due to the movement of rigid bodies relative to an observer. Optica Acta, 22, 773-791.

Koenderink, J. J. (1986). Optic flow. Vision Research, 26, 161-180. Koenderink, J. J. (1990). Solid shape. Cambridge, MA: MIT Press.

Koenderink, J. J., \& VAN Doorn, A. J. (1978). How an ambulant observer can construct a model of the environment from the geometrical structure of the visual inflow. In G. Hauske \& E. Butenandt (Eds.), Kybernetik. Munich: Springer-Verlag.

KUGLER, P. N. (1986). A morphological perspective on the origin and evolution of movement patterns. In M. G. Wade \& H. T. A. Whiting (Eds.), Motor development in children: Aspects of coordination and control (pp. 459-525). Dordrecht: Martinus Nijhoff.

KUgler, P. N., \& TURVEY, M. T. (1987). Information, natural law, and the self-assembly of rhythmic movement. Hillsdale, NJ: Erlbaum.

LAPPIN, J. S. (1990). Perceiving the metric structure of environmental objects from motion, self-motion, and stereopsis. In R. Warren \& A. H. Wertheim (Eds.), Perception and the control of self-motion (pp. 541-578). Hillsdale, NJ: Erlbaum.

LORD, E. A., \& WILSON, C. B. (1986). The mathematical description of shape and form. New York: Wiley.

MarR, D. (1982). Vision. San Francisco: W.H. Freeman.

NAKAYAMA, K. (1985). Biological image motion processing: A review. Vision Research, 25, 625-660.

NAPIER, J. (1980). Hands. New York: Pantheon.

Pittenger, J. B. (1985). Estimation of pendulum length from information in motion. Perception, 14, 247-256.

Pittenger, J. B. (1990). Detection of violations of the law of pendulum motion: Observers' sensitivity to the relation between period and length. Ecological Psychology, 2, 55-81.

QuiNLAN, P. T. (1991). Differing approaches to two-dimensional shape recognition. Psychological Bulletin, 109, 224-241.

Riccio, G. E., Martin, E. J., \& Stroffregen, T. A. (1992). The role of balance dynamics in the active perception of orientation. Journal of Experimental Psychology: Human Perception \& Performance, 18, 624-644.

Rock, I. (1973). Orientation and form. New York: Academic Press. Rock, I. (1983). The logic of perception. Cambridge, MA: MIT Press. Rock, I., DiVita, J., \& BARBeito, R. (1981). The effect on form perception of change of orientation in the third dimension. Journal of Experimental Psychology: Human Perception \& Performance, 7, 719-732.

Rock, I., \& LEAMAN, R. (1963). An experimental analysis of visual symmetry. Acta Psychologica, 21, 171-183.

Rosen, J. (1982). A symmetry primer for scientists. Cambridge: Cambridge University Press.

RUNESON, S. (1977). On the visual perception of dynamic events. Uppsala, Sweden: University of Uppsala.
Runeson, S., \& FrykHOLM, G. (1981). Visual perception of lifted weight. Journal of Experimental Psychology: Human Perception \& Performance, 7, 733-740.

Runeson, S., \& Frykholm, G. (1983). Kinematic specification of dynamics as an informational basis for person and action perception: Expectations, gender recognition, and deceptive intention. Journal of Experimental Psychology: General, 112, 585-615.

RUNESON, S., \&EDELER, D. (1993). The indispensability of precollision kinematics in the visual perception of relative mass. Perception \& Psychophysics, 53, 617-632.

Sears, F. W., Zemansky, M. W., \& Young, H. D. (1986). College physics (6th ed.). Reading, MA: Addison-Wesley.

Shaw, R. E., Mark, L. S., Jenkins, H., \& Mingolla, E. (1982), A dynamic geometry for predicting growth of gross craniofacial morphology. In Factors and mechanisms influencing bone growth (pp. 423431). New York: Liss.

Shubnikov, A. V. (1964). Colored symmetry. Oxford: Pergamon.

Solomon, Y., \& TURVEY, M. T. (1988). Haptically perceiving the distances reachable with hand-held objects. Joumal of Experimental Psychology: Human Perception \& Performance, 14, 404-427.

Solomon, Y., TURVeY, M. T., \& BURTon, G. (1989a). Gravitational and muscular variables in perceiving rod extent by wielding. Ecological Psychology, 1, 265-300.

Solomon, Y., Turvey, M. T., \& Burton, G. (1989b). Perceiving rod extents by wielding: Haptic diagonalization of the inertia tensor. Journal of Experimental Psychology: Human Perception \& Performance, $15,58-68$.

SutHERLAND, N. S. (1960). Visual discrimination of shape by octopus: Squares and rectangles. Joumal of Comparative Psychology, 53, 95-103.

SuTHERLAND, N. S. (1968). Outlines of a theory of visual pattern recognition in animals and man. Proceedings of the Royal Society of London (Series B), 171, 297-317.

Sutherland, N. S., \& CARR, A. E. (1963). The visual discrimination of shape by octopus: The effects of stimulus size. Quarterly Journal of Experimental Psychology, 13, 225-235.

THOMPSON, W. B. (1989). Structure-from-motion by tracking occlusion boundaries. Biological Cybernetics, 62, 113-116.

Thompson, W. B., MuTch, K. M., \& Berzins, V. A. (1984). Analyzing object motion based on optical flow. In Proceedings of the 7th IEEE International Conference on Pattern Recognition (pp. 791-794).

Thompson, W. B., Mutch, K. M., \& Berzins, V. A. (1985). Dynamic occlusion analysis in optical flow fields. IEEE Transactions on Pattern Analysis and Machine Intelligence, PAMI-7,4, 374-383.

TODD, J. T., \& WARREN, W. H. (1982). Visual perception of relative mass in dynamic events. Perception, 11, 325-335.

Turvey, M. T., Burton, G., Pagano, C. C., Solomon, H. Y., \& RUNESON, S. (1992). Role of the inertia tensor in perceiving object orientation by dynamic touch. Journal of Experimental Psychology: Human Perception \& Performance, 18, 714-727.

Warren, W. H., KIM, E. E., \& Husney, R. (1987). The way the ball bounces: Visual and auditory perception of elasticity and control of the bounce pass. Perception, 16, 309-336.

WAXMAN, A. M., \& UllmaN, S. (1985). Surface structure and threedimensional motion from image flow. International Journal of Robotics Research, 4, 72-94.

WeChsler, H. (1990). Computational vision. Boston, MA: Academic Press.

WEYL, H. (1921). Symmetry. Princeton, NJ: Princeton University Press. Wing, A. M. \& Fraser, C. (1983). The contribution of the thumb to reaching movements. Quarterly Journal of Experimental Psychology, 35A, 297-309.

WISER, M. (1981). The role of intrinsic axes in shape recognition. In Proceedings of the 3rd Cognitive Science Conference (pp. 184-186). San Mateo, CA: Morgan Kaufman.

\section{NOTES}

1. This is not precisely true. Fearing (1983) has shown that strict opposition of contact forces is not required. The angle between the force vectors from opposing segments of the hand can vary within a toler- 
ance determined by the frictional characteristics of the object and hand surfaces.

2 . With some misgivings, we will use "center of mass perception" as shorthand for "perception of grasp locus on an object affording a neutrally stable precision grasp" "(see Bingham \& Muchisky, 1993, for an extended discussion). The perception of this property coincides more or less with center of mass perception, but deviates from the center of mass within a tolerance determined by the frictional properties of the hand and object surfaces and by the physically determined repercussions of missing the exact locus of the center of mass. For purposes of measurement, the frictional component of grasping was not emphasized in our studies.

3. Without vision, the object was typically grasped at an arbitrary point of first contact and lifted slightly to obtain haptic information. The grasp was then moved to the desired location, which was the center of mass in such appropriate instances as that of the crank handle.
4. Use of intrinsic geometries would obviate this requirement (Koenderink, 1990; Koenderink \& van Doorn, 1978; Lappin, 1990; Lord \& Wilson, 1986).

5. An exception is that of rotations that preserve reflective symmetry (Deutsch, 1955).

6. The center of area is the first moment of a plane figure and is the same as the center of mass of a planar object of the same shape with constant mass per unit area. Area is the zeroth moment, while the moment of inertia is the second moment. Use of moments in computing shape is standard (Ballard \& Brown, 1982; Horn, 1986), but not universal (Wechsler, 1990).

(Manuscript received April 10, 1992; revision accepted for publication April 9, 1993.) 